Svetina, C., Mankowsky, R., Knopp, G., Koch, F., Seniutinas, G., Rösner, B., ... Beaud, P. (2019). Towards X-ray transient grating spectroscopy. Optics Letters, 44(3), 574-577.

https://doi .org/10.1364/oL.44.000574

\title{
Towards X-ray Transient Grating Spectroscopy
}

\author{
C. Svetina, ${ }^{1,{ }^{*}}$ R. Mankowsky, ${ }^{1}$ G. KnOpp, ${ }^{1}$ F. KOCH, ${ }^{1}$ G. Seniutinas, ${ }^{1}$ B. \\ Rösner, ${ }^{1}$ A. Kubec, $^{1}$ M. Lebugle, ${ }^{1}$ I. Mochi, ${ }^{1}$ M. BeCK, ${ }^{1}$ C. Cirelli, ${ }^{1}$ J. \\ Krempasky, ${ }^{1}$ C. Pradervand, ${ }^{1}$ J. Rouxel, ${ }^{1,2}$ G. Mancini, ${ }^{1,2}$ S. Zerdane, ${ }^{1}$ B. \\ Pedrini, ${ }^{1}$ V. EsPosito, ${ }^{1}$ G. INGOLd, ${ }^{1}$ U. WAgner, ${ }^{1}$ U. FleChSIG, ${ }^{1}$ R. FOLlath, ${ }^{1}$ \\ M. Chergui, ${ }^{2}$ C. Milne, ${ }^{1}$ H.T. Lemke, ${ }^{1}$ C. David ${ }^{1}$ And P. Beaud ${ }^{1}$ \\ ${ }^{1}$ Paul Scherrer Institut, 5232 Villigen PSI, Switzerland \\ ${ }^{2}$ Ecole Polytechnique Fédérale de Lausanne, Laboratoire de Spectroscopie UItrarapide and Lausanne Centre for UItrafast Science, ISIC, Station 6, \\ 1015 Lausanne, Switzerland \\ *Corresponding author: cristian.svetina@psi.ch
}

Received XX Month XXXX; revised XX Month, XXXX; accepted XX Month XXXX; posted XX Month XXXX (Doc. ID XXXXX); published XX Month XXXX

The extension of transient grating spectroscopy (TGS) to the $x$-ray regime will create numerous opportunities ranging from the study of thermal transport in the ballistic regime to charge, spin and energy transfer processes with atomic spatial and femtosecond temporal resolution. Studies involving complicated split-and-delay lines have not yet been successful in achieving this goal. Here, we propose a novel, simple method based on the Talbot effect for converging beams, which can easily be implemented at current X-ray Free Electron Lasers. We validate our proposal by analyzing printed interference patterns on PMMA and Gold samples using $~ 3 \mathrm{keV} X$-ray pulses.

OCIS codes: (190.4380) Nonlinear optics; (140.2600) Free-electron lasers (FELs); (340.7480) X-rays, soft X-rays, extreme ultraviolet (EUV).

http://dx.doi.org/10.1364/OL.99.099999

Transient grating spectroscopy (TGS) is a well-established technique in the field of nonlinear spectroscopy $[1,2]$ to study electronic energy transport, diffusion and coherent dynamics in crystalline, amorphous, liquid and gaseous materials [3-6]. The transient grating is generated by two identical laser pulses, which are overlapped on the sample in space and time, generating an interference pattern with period $\Lambda=\lambda /(2 \sin (\vartheta / 2))$ defined by the wavelength $\lambda$ and the angle $\vartheta$ between the two beams. This pattern is transferred into the sample as the light is absorbed, creating a periodically modulated excitation that subsequently evolves in time and eventually decays. These dynamics can be investigated by probing the transient grating with a third pulse and monitoring its diffraction intensity for different arrival time delays. This measurement is sensitive to diffusion dynamics and coherent excitations with spatial resolution $\Lambda$ and a momentum transfer $q=2 \pi / \Lambda$, given by the momentum conservation of the four pulses (three incoming beams, one outgoing detected beam).

TGS has been developed using table-top lasers with wavelengths ranging from infrared (IR) to ultraviolet (UV) [7, 8]. More recently, the technique has been successfully demonstrated in the EUV range at the FERMI Free-Electron Laser (FEL), where the TIMER beamline has now been fully dedicated to TGS and to four-wave mixing techniques (FWM) in general [9-12]. While this outstanding technological development increased the accessible momentum transfer and spatial resolution (down to tens of $\mathrm{nm}$ ), measurements with light in the EUV range are generally limited to surface properties due strong absorption and diffusion processes across single or few unit cells are still inaccessible.

These limitations can be overcome by transferring the technique to X-ray FELs. X-ray TGS can be used for example to study ballistic thermal transport, an important research area driven by relevant technological needs such as thermal management of electronic devices at the nanoscale [13-15], where the conventional heat diffusion theory fails [16]. More importantly, the relatively low absorption at X-ray photon energies allows the study of the bulk properties of the investigated materials. By tuning the X-ray energy to specific atomic transitions, FEL sources offer the possibility of elemental and site specific excitations [17]. Moreover, recent theoretical studies propose application of soft and hard X-ray FWM techniques to investigate charge, spin and energy transfer processes as well as exciton dynamics in strongly correlated materials with atomic spatial and femtosecond temporal resolution [18-20].

Efforts to extend TGS into the X-ray regime, based on complicated X-ray split and delay lines, have not yet been successful. Here, we propose a novel, simpler approach based on a transmission grating as a single optical element to create spatial excitation profiles in the soft and hard X-ray regimes. We experimentally demonstrate the grating generation using X-ray 
pulses at a wavelength of $\lambda \sim 4 \AA$ and validate the presented method by analyzing permanent imprints in gold and spin-coated polymethyl methacrylate (PMMA) generated at X-ray fluences exceeding the materials' damage threshold.

In our optical scheme we take advantage of the Talbot effect generated by a 1D diffraction phase grating. A plane wave propagating through a grating is diffracted into several orders (Fig.1a). The superposition of these diffracted beams creates replicas of the diffraction grating at evenly-spaced distances that are named Talbot planes [21]. The distance $D_{\mathrm{n}}$ of the Talbot planes as well as the period of the fringe pattern are a function of $\lambda$ and of the pitch $p$ of the diffraction grating:

$$
D_{n}=\frac{1}{\eta^{2}} \frac{n p^{2}}{2 \lambda},
$$

where $\mathrm{n}$ is an integer called the Talbot order and $\eta$ is a parameter equal to 1 for an amplitude or $\pi / 2$ phase grating , and equal to 2 for a $\pi$ phase grating. The fringe spacing is equal to the grating's pitch $p$ for amplitude and $\pi / 2$ phase gratings while it is $p / 2$ for $\pi$ phase gratings [22]. When the incoming wave is divergent the Talbot planes are further and further separated and the replicas have a larger fringe spacing than the diffraction grating. In this case the Talbot distances become $d_{n}=$ $L D_{n} /\left(L-D_{n}\right)$, where $L$ is the distance between the focus and the diffraction grating. Here the fringe's spacing is multiplied by the magnification factor $M=\left(L+D_{n}\right) / L$. This effect is widely used in differential phase contrast X-ray imaging [23-25], interferometry [26-28] as well as tomography [29] and is the basic principle of a class of X-ray wave-front sensors [30,31].
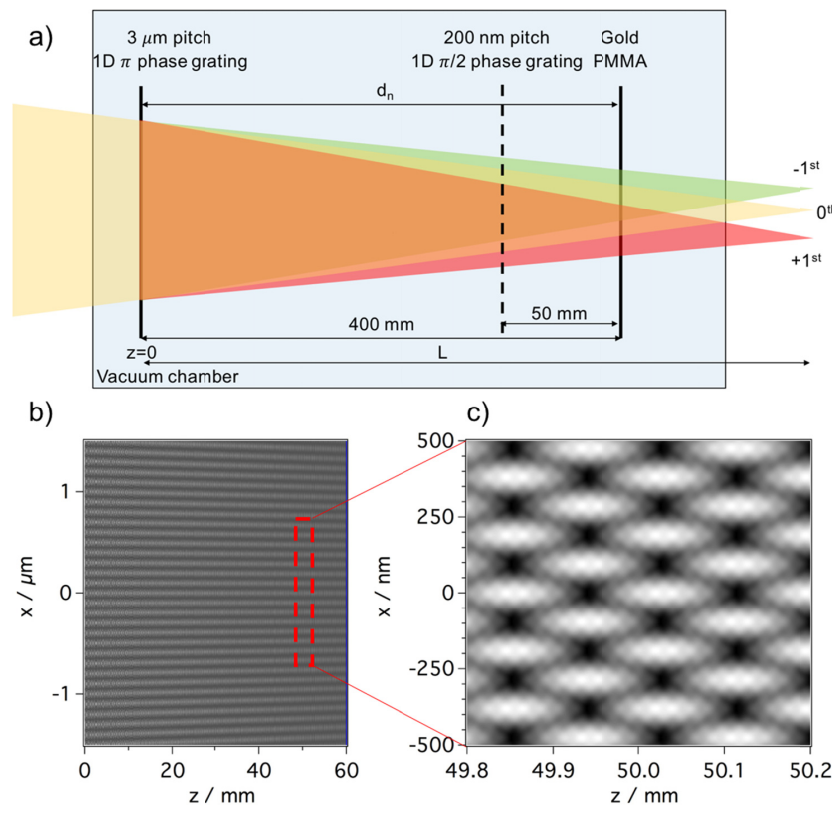

b)

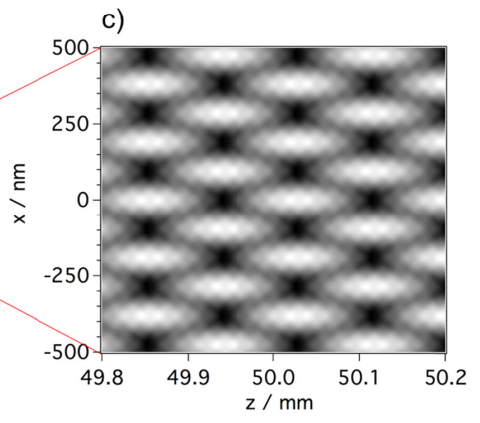

Fig. 1. (a) Layout depicting the experimental conditions at the Alvra experimental station. (b) Fresnel simulation of the Talbot carpet: intensity distribution of the experimental conditions for a 1D diamond phase grating with $200 \mathrm{~nm}$ pitch and $2.985 \mathrm{keV}$ photon energy. The inset (c) shows the detailed structure of the interference pattern in the vicinity of the sample where the pitch is $190 \mathrm{~nm}$.
Here, we go the opposite way and combine the Talbot effect with a convergent beam. In this case, the Talbot planes move closer together and the fringe's spacing gets smaller approaching the focus. Fresnel propagation simulations using the experimental conditions are shown in Figure 1b. For a photon energy of 2.985 $\mathrm{keV}$ and a $200 \mathrm{~nm}$ pitch $1 \mathrm{D} \pi / 2$ phase grating placed $50 \mathrm{~mm}$ upstream of the sample, calculations predict a projected grating with $189 \mathrm{~nm}$ pitch on the sample.

To validate this method experimentally, we imprinted gratings on gold and PMMA samples. The measurements have been carried out at the Alvra experimental station at the Swiss X-ray FreeElectron Laser SwissFEL [32]. The X-ray pulses used for this experiment had a bandwidth of $15-20 \mathrm{eV}$ and an estimated pulse duration of $50 \mathrm{fs}$ rms. All measurements were done at constant Xray pulse energy of $125 \mu \mathrm{J}$. The experiment was performed with two 1D diamond diffraction gratings [33], as shown in Figure 2. First, a coarse $\pi$ phase-shift grating with a pitch of $3 \mu \mathrm{m}$ was placed $400 \mathrm{~mm}$ upstream of the sample to create imprints with a pitch of $1 \mu \mathrm{m}$ for alignment purposes and $\mathrm{L}=1.40 \mathrm{~m}$. Subsequently, a $\pi / 2$ phase grating with a pitch of $200 \mathrm{~nm}$ was placed $50 \mathrm{~mm}$ upstream of the sample to generate fine imprints on the nanometer scale with $\mathrm{L}=1.05 \mathrm{~m}$. The gratings and the targets were placed in vacuum to minimize air absorption losses. The beam footprint on the target was set to $400 \mu \mathrm{m}$ horizontally and $200 \mu \mathrm{m}$ vertically full-width-half-maximum (FWHM) using the beamline focusing system, composed of a pair of bendable Kirkpatrick-Baez (KB) mirrors [34]. For the given FEL power no visible sign of damage could be found on the diffraction gratings.

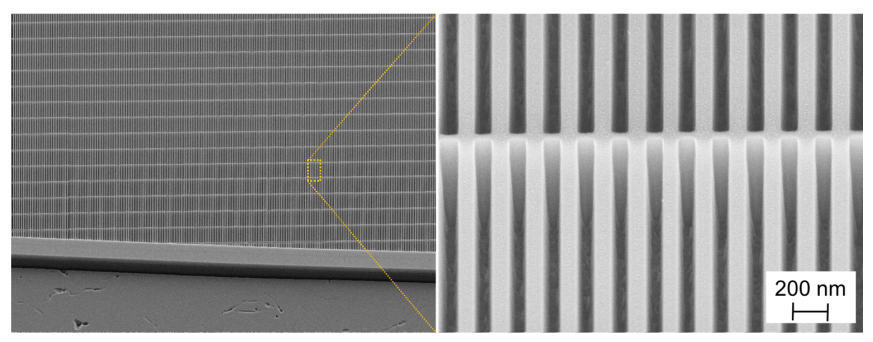

Fig. 2. Scanning electron microscope (SEM) images of the $200 \mathrm{~nm}$ pitch 1D diamond grating.

During the experiment, the investigated targets have been exposed to both single and multiple pulses using a fast shutter. Printing on gold at $3 \mathrm{keV}$ is rather easy due to the small attenuation length $(250 \mathrm{~nm})$ while PMMA $(67.4 \mu \mathrm{m})$ requires significantly higher fluences to be patterned. Considering the overall beamline transmission, the grating efficiencies for the first diffraction orders (39\% for the $3 \mu \mathrm{m}$ pitch and $8 \%$ for the $200 \mathrm{~nm}$ period) and an aspect ratio of 0.5 , the fluences within the interference pattern are estimated to be around $90 \mathrm{~mJ} / \mathrm{cm}^{2}$ and $25 \mathrm{~mJ} / \mathrm{cm}^{2}$ for the coarse and fine diffraction gratings respectively.

The imprints (Fig.3) produced by the coarse $3 \mu \mathrm{m}$ pitch grating have been inspected by means of optical microscopy, scanning electron microscopy (SEM) and atomic force microscopy (AFM). We have found single shot imprinted grating structures on both samples with a period of about $0.95 \mu \mathrm{m}$, even without development of the PMMA [35]. The fluence was above the ablation threshold of both materials. The printed patterns on gold shows an uneven modulation of the intensity due to the fact that the sample was not perfectly sitting on the Talbot plane. 
The imprints generated by the $200 \mathrm{~nm}$ pitch $\pi / 2$ phase grating have been investigated with SEM and AFM on both samples. We found clear signatures of gratings generated from single shots with a periodicity of $185.7 \mathrm{~nm}$ on PMMA and $187.7 \mathrm{~nm}(+/-0.3 \mathrm{~nm})$ on Gold (Fig. 4), in very good agreement with the value of $189 \mathrm{~nm}$ obtained from the simulations. The 1000 shots reference imprint shows a printed grating with a pitch of $188.5 \mathrm{~nm}$, demonstrating not only the excellent pointing stability of the FEL but also the feasibility of experiments, which require integration over multiple shots.

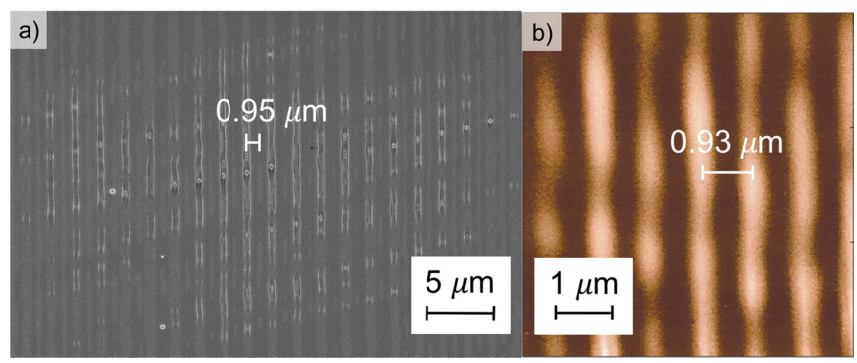

Fig. 3. SEM image on gold (a) and AFM image on PMMA (b) from a single X-ray pulse using the $3 \mu \mathrm{m}$ pitch grating.

In summary, we have proposed a simple experimental configuration based on a single optical element to generate transient gratings in the soft and hard X-ray regime that can easily be applied at most beamlines of current XFELs. This technique takes advantage of a 1D phase grating in conjunction with the Talbot effect and convergent beams to create demagnified replica of the diffraction grating. We have confirmed our model calculations and validated the suitability of this technique by imprinting permanent gratings on gold and PMMA test targets using ultrashort X-ray pulses at a photon energy of $3 \mathrm{keV}$ generated at an FEL. The generation of transient gratings for repetitive pump-probe experiments simply requires a reduction of the X-ray fluence below the damage threshold.

The magnification factor achieved in our experiment was only 0.95 , i.e. a $5 \%$ decrease of the original phase gratings pitch. We want to emphasize that smaller values can easily be achieved by moving the X-ray focus closer to the sample, which however also reduces the spot size on the sample. Changing the grating pitch by moving the focus can further be used to perform $\mathbf{q}$-scans at fixed time delays.

The achromatic Talbot effect can be used to generate gratings with even smaller pitch [36-38]. Using the full bandwidth of the FEL X-ray pulses and a proper geometry, the self-replicas of the phase grating will merge into a continuous grating along the direction of propagation while the periodicity becomes half that of the original grating's pitch. This, combined with a strong focusing would allow the creation of XTGs on the order of tens of nanometers.

The proposed method can be implemented at any X-FEL and opens a new scientific perspective to extend TGS to energy and length scales so far inaccessible, offering the ability to probe a variety of dynamical processes, from acoustic phonons to heat transfer, at the nanoscale.
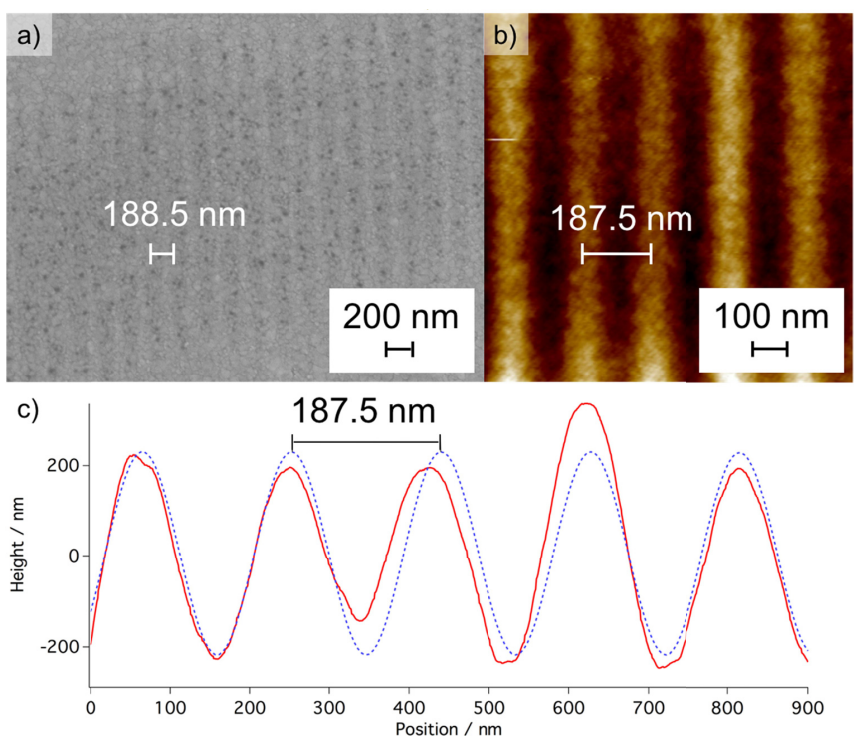

Fig. 4. SEM image on gold (a) and AFM image on PMMA (b) of the printed patterns. Line profile of the AFM image and sinusoidal fit showing the periodicity of the printed grating (c).

Funding. NCCR Molecular Ultrafast Science and Technology (NCCR MUST), a research instrument of the Swiss National Science Foundation (SNSF). European Union's Horizon 2020 Research and Innovation programme (Grant Agreement 730872). European Research Council Advanced Grant H2020 ERCEA (695197 DYNAMOX).

Acknowledgment. We are grateful to Claudio Masciovecchio for fruitful discussions.

\section{References}

1. Y. Shen, Principles of Nonlinear Optics, John Wiley \& Sons, New York, (1984).

2. N. Bloembergen, Rev. Mod. Phys. 54, 685 (1982).

3. A. Taschin, R. Eramo, P. Bartolini, and R. Torre, Time-Resolved Spectroscopy of Complex Liquids, Springer, New York (2008).

4. H. Eichler (Ed.), Special issue on dynamic grating and four-wave mixing, IEEE J. Quant. Electron. QE-22 (1986).

5. S. Tanaka and S. Mukamel, Phys. Rev. Lett. 89, 043001 (2002).

6. S.T. Cundiff and S. Mukamel, Physics Today 66 (7), 44 (2013).

7. L. Dhar, J. A. Rogers and K. A. Nelson, Chem. Rev. 94, 157 (1994).

8. A. Ajdarzadeh, C. Consani, O. Bram, A. Tortschanoff, A. Cannizzo and M. Chergui, Chemical Physics 422 (2013).

9. R. Cucini, F. Bencivenga and C. Masciovecchio, Opt. Lett., 36, 1032 (2011).

10. F. Bencivenga, S. Baroni, C. Carbone, M. Chergui, M. B. Danailov, G. De Ninno, M. Kiskinova, L. Raimondi, C. Svetina and C. Masciovecchio, New J. Phys. 15123023 (2013).

11. F. Bencivenga, R. Cucini, F. Capotondi, A. Battistoni, R. Mincigrucci, E. Giangrisostomi, A. Gessini, M. Manfredda, I. P. Nikolov, E. Pedersoli, E. Principi, C. Svetina, P. Parisse, F. Casolari, M. B. Danailov, M. Kiskinova and C. Masciovecchio, Nature 520, 205 (2015).

12. L. Foglia, F. Capotondi, R. Mincigrucci, D. Naumenko, E. Pedersoli, A. Simoncig, G. Kurdi, A. Calvi, M. Manfredda, L. Raimondi, N. Mahne, M. Zangrando, C. Masciovecchio, and F. Bencivenga, Phys. Rev. Lett. 120, 263901 (2018).

13. M. E. Siemens, Q. Li, R. Yang, K. A. Nelson, E. H. Anderson, M. M. Murnane and H. C. Kapteyn, Nat. Mater. 9, 26 (2010). 
14. D. G. Cahill, W. K. Ford, K. E. Goodson, G. D. Mahan, A. Majumdar, H. J. Maris, R. Merlin and S. R. Phillpot, J. Appl. Phys. 93, 793 (2003).

15. E. Pop, S. Sinha and K. E. Goodson, Proc. of the IEEE, Vol. 94, No. 8, (2006).

16. A. Dhar, Adv. Phys., 57, 457 (2008).

17. J. Stöhr, NEXAFS Spectroscopy, Springer (1996).

18. S. Mukamel, D. Healion, Y. Zhang and J. D. Biggs, Ann. Rev. Phys. Chem 64, 101 (2013).

19. S. Mukamel, D. Abramavicius, L. Yang, W. Zhuang, I. V. Schweigert and D. V. Voronine, Acc. Chem. Res. 42 (4), 553 (2009).

20. J. D. Biggs, Y. Zhang, D. Healion and S. Mukamel, J. Chem. Phys, 136, 174117 (2012).

21. H. F. Talbot, Facts relating to optical science IV, Phil. Mag. 9, (1836).

22. T. Weitkamp, C. David, C. Kottler, O. Bunk and F. Pfeiffer., Proc. SPIE 6318, 63180S-1 (2006).

23. M. Engelhardt, J. Baumann, M. Schuster, C. Kottler, F. Pfeiffer, O. Bunk and C. David, Appl. Phys. Lett. 90, 224101 (2007).

24. H. H. Wen, E. E. Bennett, R. Kopace, A. F. Stein and V. Pai, Opt. Lett. 35, 1932 (2010).

25. C. David, B. Nohammer and H. H. Solak, Appl. Phys. Lett. 81, 3287, (2002).

26. A. W. Lohmann, D. E. Silva, Opt. Commun. 2, 413 (1971).

27. A. Momose, S. Kawamoto, I. Koyama, Y. Hamaishi, K. Takai and Y. Suzuki, Jpn. J. Appl. Phys. 42, L866 (2003).

28. T. Weitkamp, I. Zanette, C. David, J. Baruchel, M. Bech, P. Bernard, H. Deyhle, T. Donath, J. Kenntner S. Lang, J. Mohr, B. Müller, F. Pfeiffer, E. Reznikova, S. Rutishauser, G. Schulz, A. Tapfer and J.P. Valade, Proc. SPIE 7804, 780406-1 (2010).

29. C. David, T. Weitkamp, F. Pfeiffer, A. Diaz, J. Bruder, T. Rohbeck, A. Groso, O. Bunk, M. Stampanoni and P. Cloetens, Spectrochim. Acta B 62, 626 (2007).

30. S. Rutishauser, L. Samoylova, J. Krzywinski, O. Bunk, J. Grünert, H. Sinn, M. Cammarata, D. M. Fritz and C. David, Nat. Commun. 3, 947 (2012).

31. T. Weitkamp, B. Nohammer, A. Diaz and C. David, Appl. Phys. Lett. 86, 054101 (2005).

32. C. J. Milne, T. Schietinger, M. Aiba, A. Alarcon, J. Alex, A. Anghel, V. Arsov, C. Beard, P. Beaud, et al., Appl. Sci. 7, 720 (2017).

33. M. Makita, P. Karvinen, V. A. Guzenko, N.Kujala, P- Vagovic and C. David, Microelectron. Eng. 176, 75 (2017).

34. P. Kirkpatric, A. V. Baez, J. Opt. Soc. Am. 38, 766 (1948).

35. B. Rösner, F. Doring, P. R. Ribic, D. Gauthier, E. Principi, C. Masciovecchio, M. Zangrando, J. V. Comamala, G. De Ninno and C. David, Opti. Express 25, 30686 (2017).

36. D. Fan, E. Buitrago, S. Yang, W. Karim, Y. Wu, R. Tai and Y. Ekinci, Microelectron. Eng. 155, 55 (2016).

37. . Danylyuk, P. Loosen, K. Bergmann, H. Kim and L. Juschkin, J. Micro. Nanolith. MEMS MOEMS 12, 033002 (2013).

38. J. Rizzi, T. Weitkamp, N. Guerineau, M. Idir, P. Mercere, G. Druart, G. Vincent, P. da Silva and J. Primot, Opt. Lett. 36, 1398 (2011) 


\section{References}

1. Y. Shen, Principles of Nonlinear Optics, John Wiley \& Sons, New York, (1984).

2. N. Bloembergen, "Nonlinear optics and spectroscopy", Rev. Mod. Phys. 54 (3), 685-695 (1982).

3. A. Taschin, R. Eramo, P. Bartolini, and R. Torre, Time-Resolved Spectroscopy in Complex Liquids, Springer, New York (2008).

4. H. Eichler (Ed.), Special issue on dynamic grating and four-wave mixing, IEEE J. Quant. Electron. QE-22 (1986).

5. S. Tanaka and S. Mukamel, "Coherent X-Ray Raman Spectroscopy: A Nonlinear Local Probe for Electronic Excitations”, Phys. Rev. Lett. 89, 043001 (2002).

6. S.T. Cundiff and S. Mukamel, “Optical multidimensional coherent spectroscopy”, Physics Today 66 (7), 44 (2013).

7. L. Dhar, J. A. Rogers and K. A. Nelson, "Time-resolved vibrational spectroscopy in the impulsive limit", Chem. Rev. 94 (1), 157-193 (1994).

8. A. Ajdarzadeh, C. Consani, O. Bram, A. Tortschanoff, A. Cannizzo and M. Chergui, "Ultraviolet transient absorption, transient grating and photon echo studies of acqueous tryptophan", Chemical Physics 422, 47-52 (2013).

9. R. Cucini, F. Bencivenga and C. Masciovecchio, "All-reflective femtosecond optical pump-probe setup for transient grating spectroscopy", Opt. Lett., 36 (7), 1032-1034 (2011).

10. F. Bencivenga, S. Baroni, C. Carbone, M. Chergui, M. B. Danailov, G. De Ninno, M. Kiskinova, L. Raimondi, C. Svetina and C. Masciovecchio, "Nanoscale dynamics by short-wavelength four wave mixing experiments", New J. Phys. 15123023 (2013).

11. F. Bencivenga, R. Cucini, F. Capotondi, A. Battistoni, R. Mincigrucci, E. Giangrisostomi, A. Gessini, M. Manfredda, I. P. Nikolov, E. Pedersoli, E. Principi, C. Svetina, P. Parisse, F. Casolari, M. B. Danailov, M. Kiskinova and C. Masciovecchio, "Four-wave mixing experiments with extreme ultraviolet transient gratings", Nature 520, 205-208 (2015).

12. L. Foglia, F. Capotondi, R. Mincigrucci, D. Naumenko, E. Pedersoli, A. Simoncig, G. Kurdi, A. Calvi, M. Manfredda, L. Raimondi, N. Mahne, M. Zangrando, C. Masciovecchio, and F. Bencivenga, "First Evidence of Purely Extreme-Ultraviolet Four-Wave Mixing”, Phys. Rev. Lett. 120, 263901 (2018).

13. M. E. Siemens, Q. Li, R. Yang, K. A. Nelson, E. H. Anderson, M. M. Murnane and H. C. Kapteyn, “'”, Nat. Mater. 9 (1), 26-30 (2010).

14. D. G. Cahill, W. K. Ford, K. E. Goodson, G. D. Mahan, A. Majumdar, H. J. Maris, R. Merlin and S. R. Phillpot, "Nanoscale thermal transport", J. Appl. Phys. 93, 793-818 (2003).

15. E. Pop, S. Sinha and K. E. Goodson, "Heat Generation and Transport in Nanometer-Scale Transistors", Proc. of the IEEE, Vol. 94, No. 8, 1587-1601 (2006).

16. A. Dhar, "Heat transport in low-dimensional systems", Adv. Phys., 57 (5), 457-537 (2008).

17. J. Stöhr, NEXAFS Spectroscopy, Springer (1996).

18. S. Mukamel, D. Healion, Y. Zhang and J. D. Biggs, "Multidimensional Attosecond Resonant X-Ray Spectroscopy of Molecules: Lessons from the Optical Regime", Ann. Rev. Phys. Chem 64, 101-127 (2013).

19. S. Mukamel, D. Abramavicius, L. Yang, W. Zhuang, I. V. Schweigert and D. V. Voronine, "Coherent Multidimensional Optical Probes for Electron Correlations and Exciton Dynamics: From NMR to X-rays", Acc. Chem. Res. 42 (4), 553-562 (2009).

20. J. D. Biggs, Y. Zhang, D. Healion and S. Mukamel, "Two-dimensional stimulated resonance Raman spectroscopy of molecules with broadband x-ray pulses", J. Chem. Phys, 136 (17), 174117 (2012).

21. H. F. Talbot, "Facts relating to optical science. No. IV", Phil. Mag. 9, 401407 (1836).

22. T. Weitkamp, C. David, C. Kottler, O. Bunk and F. Pfeiffer., "Tomography with grating interferometers at low-brilliance sources", Proc. SPIE 6318, 63180S-1 (2006).
23. M. Engelhardt, J. Baumann, M. Schuster, C. Kottler, F. Pfeiffer, O. Bunk and C. David, "High-resolution differential phase contrast imaging using a magnifying projection geometry with a microfocus x-ray source", Appl. Phys. Lett. 90 (22), 224101 (2007).

24. H. H. Wen, E. E. Bennett, R. Kopace, A. F. Stein and V. Pai, "Single-shot Xray differential phase-contrast and diffraction imaging using twodimensional transmission gratings", Opt. Lett. 35 (12), 1932-1934 (2010).

25. C. David, B. Nohammer and H. H. Solak, "Differential x-ray phase contrast imaging using a shearing interferometer", Appl. Phys. Lett. 81 (17), 32873289 (2002).

26. A. W. Lohmann, D. E. Silva, "An interferometer based on the Talbot effect", Opt. Commun. 2 (9), 413-415 (1971).

27. A. Momose, S. Kawamoto, I. Koyama, Y. Hamaishi, K. Takai and Y. Suzuki, "Demonstration of X-Ray Talbot Interferometry", Jpn. J. Appl. Phys. 42, L866 (2003).

28. T. Weitkamp, I. Zanette, C. David, J. Baruchel, M. Bech, P. Bernard, H. Deyhle, T. Donath, J. Kenntner S. Lang, J. Mohr, B. Müller, F. Pfeiffer, E. Reznikova, S. Rutishauser, G. Schulz, A. Tapfer and J.P. Valade, "Recent developments in x-ray Talbot interferometry at ESRF-ID19", Proc. SPIE 7804, 780406-1 (2010).

29. C. David, T. Weitkamp, F. Pfeiffer, A. Diaz, J. Bruder, T. Rohbeck, A. Groso, O. Bunk, M. Stampanoni and P. Cloetens, "Hard X-ray phase imaging and tomography using a grating interferometer", Spectrochim. Acta B 62 (6-7), 626-630 (2007).

30. S. Rutishauser, L. Samoylova, J. Krzywinski, O. Bunk, J. Grünert, H. Sinn, M. Cammarata, D. M. Fritz and C. David, "Exploring the wavefront of hard Xray free-electron laser radiation", Nat. Commun. 3, 947 (2012).

31. T. Weitkamp, B. Nohammer, A. Diaz and C. David, "X-ray wavefront analysis and optics characterization with a grating interferometer", Appl. Phys. Lett. 86 (5), 054101 (2005).

32. C. J. Milne, T. Schietinger, M. Aiba, A. Alarcon, J. Alex, A. Anghel , V. Arsov, C. Beard, P. Beaud, et al., "SwissFEL: The Swiss X-ray Free Electron Laser", Appl. Sci. 7 (7), 720 (2017).

33. M. Makita, P. Karvinen, V. A. Guzenko, N.Kujala, P- Vagovic and C. David, "Fabrication of diamond diffraction gratings for experiments with intense hard x-rays", Microelectron. Eng. 176, 75-78 (2017).

34. P. Kirkpatrick, A. V. Baez, "Formation of Optical Images by X-Rays", J. Opt. Soc. Am. 38 (9), 766-774 (1948).

35. B. Rösner, F. Doring, P. R. Ribic, D. Gauthier, E. Principi, C. Masciovecchio, M. Zangrando, J. V. Comamala, G. De Ninno and C. David, "High resolution beam profiling of $\mathrm{X}$-ray free electron laser radiation by polymer imprint development", Opti. Express 25 (24), 30686-30695 (2017).

36. D. Fan, E. Buitrago, S. Yang, W. Karim, Y. Wu, R. Tai and Y. Ekinci, "Patterning of nanodot-arrays using EUV achromatic Talbot lithography at the Swiss Light Source and Shanghai Synchrotron Radiation Facility", Microelectron. Eng. 155, 55-60 (2016).

37. S. Danylyuk, P. Loosen, K. Bergmann, H. Kim and L. Juschkin, "Scalability limits of Talbot lithography with plasma-based extreme ultraviolet sources", J. Micro. Nanolith. MEMS MOEMS 12 (3), 033002 (2013).

38. J. Rizzi, T. Weitkamp, N. Guerineau, M. Idir, P. Mercere, G. Druart, G. Vincent, P. da Silva and J. Primot, "Quadriwave lateral shearing interferometry in an achromatic and continuously self-imaging regime for future x-ray phase imaging", Opt. Lett. 36 (8), 1398-1400 (2011). 